\title{
SÍNDROME ANTIFOSFOLÍPIDOS Y TROMBOCITOPENIA MATERNA EN GEMELAR BICORIÓNICO CON RESTRICCIÓN FETAL GRAVE
}

\author{
Jorge Hasbun H. ${ }^{1}$, Guillermo Conte L. ${ }^{1}$, Waldo Sepúlveda L. ${ }^{2}$, María Angélica Manrí- \\ quez S. ${ }^{3}$, José Martínez M. ${ }^{2}$ \\ ${ }^{1}$ Departamento de Obstetricia y Ginecología, Hospital Clínico de la Universidad de Chile. ${ }^{2}$ Departamento de Obstetricia \\ y Ginecología, Clínica Las Condes. ${ }^{3}$ Laboratorio Histopatología Citolab.
}

\section{RESUMEN}

Se presenta un caso clínico de síndrome antifosfolípidos con antecedente de mortinato y tratamiento anticoagulante prolongado. En la segunda gestación (gemelar bicoriónico) desarrolla una restricción fetal precoz selectiva en un gemelo, seguido de detención definitiva de su crecimiento en semana 25. Luego del tratamiento de una trombocitopenia materna intercurrente se decidió la interrupción del embarazo en semana 28 por operación cesárea. Ambos gemelos de 860 y 406 gramos sobrevivieron en buenas condiciones al período neonatal. Se discuten los mecanismos etiopatogénicos y aspectos singulares observados en la adaptación circulatoria del gemelo B.

PALABRAS CLAVES: Síndrome antifosfolípidos, gemelar bicoriónico, trombocitopenia, restricción del crecimiento fetal

\section{SUMMARY}

We report a clinical case of antiphospholipid syndrome with previous fetal death and extended anticoagulant treatment. At a second gestation (bichorionic twins) an early selective restriction of one fetus was developed which stopped growth on week 25. After a treated maternal thrombocytopenia we proceed with an elective premature delivery by cesarean section on week 28. Both twins ( 860 and 406 grams each) survived the neonatal period. We discuss the ethiopatogenic probable mechanisms and singular aspects of circulatory adaptation of twin $B$ in a very complex case and management.

\section{KEY WORDS: Antiphospholipid syndrome, bichorionic twins, thrombocytopenia, fetal growth restriction}

\section{INTRODUCCIÓN}

El síndrome antifosfolípidos (SAF) se define por la presencia de anticuerpos antifosfolípidos (AAF), trombosis arterial ó venosa recurrente, abortos espontáneos y ocasionalmente trombocitopenia autoinmune (1). Es la causa más frecuente de trombofilia adquirida con manifestaciones clínicas trombóticas en cualquier territorio vascular (2).
Hay descritos casos primarios catastróficos, con microangiopatía trombótica y falla multisistémica, confirmando su heterogeneidad y la importancia de la pesquisa y manejo precoces $(3,4,5,6)$.

El diagnóstico clínico se plantea ante una trombosis asociada a anticoagulante lúpico persistente y/o presencia de niveles elevados de anticuerpos anticardiolipinas (IgG, $\lg M, \lg A)$. Los antígenos blancos de los AAF están dirigidos a cofactores 
protéicos unidos a fosfolípidos, principalmente Beta 2 glicoproteina I (B2GPI), y/o anticuerpos contra complejos de proteínas unidas a fosfolípidos 0 solo a fosfolípidos (7).

EI SAF se ha clasificado en cinco grupos: autoinmune primario o secundario a lupus eritematoso diseminado (LES), asociado a infecciones, cáncer, medicamentos o presente en personas sanas de la población general (1 a $5 \%$ ).

Normalmente los fosfolípidos de células endoteliales están unidos a proteínas de enlace que al desencadenarse un SAF pierden su capacidad de unión y son reemplazadas por los AAF. Sus mecanismos son diferentes, por interferencia en los sistemas de anticoagulantes endógenos, en endotelio vascular, en plaquetas, en leucocitos y en la fibrinolisis $(7,8)$.

En embarazadas la prevalencia de complicaciones es de 15 a $20 \%$, puede haber preeclampsia precoz, restricción grave del crecimiento fetal, prematurez, óbito fetal $(9,10)$. En su etiopatogenia hay un rol de los anticuerpos sobre la coagulación generando trombosis y sobre el trofoblasto limitando su desarrollo $(11,12)$.

Presentamos un caso clínico de SAF con tratamiento anticoagulante de 5 años de evolución, que en el segundo embarazo desarrolla complicaciones materno-fetales infrecuentes, de alto riesgo, generando decisiones complejas de tratamiento que justifican su comunicación.

\section{CASO CLÍNICO}

Paciente de 32 años, gesta 2, para 1. En su primer embarazo en semana 30 presentó preeclampsia y óbito fetal de 1.100 gramos sin mal-

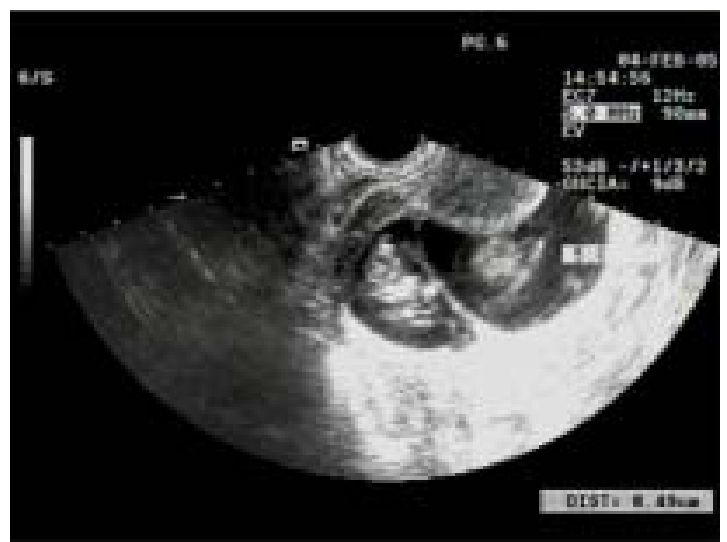

Figura 1. Ultrasonido en semana 12 con asimetría de sacos. formaciones, trombocitopenia de 74.000, TTPK 51 segundos, monómero de fibrina en red $(+)$, transaminasas de $46(\mathrm{OA})$ y $53(\mathrm{P})$, recuperándose sin incidentes. La histomorfología placentaria demostró infartos antiguos y lesiones vellositarias secundarias a preeclampsia. El estudio materno confirmó SAF con presencia de anticoagulante lúpico y títulos altos persistentes de AAF iniciando anticoagulación oral (acenocumarol) mantenida por cinco años, con INR entre 2,0-3,0 agregándose enalapril en últimos dos años por hipertensión crónica moderada.

Embarazo actual: concepción el 24 noviembre 2004, confirmada la actividad cardiaca embrionaria se cambia anticoagulación oral a heparina de bajo peso molecular (HBPM) $5000 \mathrm{U}$ sbc/día e hipotensor a alfa-metildopa $375 \mathrm{mg} /$ día asociando aspirina (ASA) $100 \mathrm{mg} /$ día (semana 12). En semana 7 el ultrasonido (US) demostró gemelar bicoriónicobiamniótico con discordancia precoz en el tamaño de los sacos, confirmada en semana 12 (Figura 1). En semana 19 hay discordancia en biometría y peso con flujo diastólico presente en arteria umbilical del gemelo restringido (Figura 2), sin embargo el flujo de ambas arterias uterinas se mantiene normal. En semana 20 el estudio genético por amniocentesis demostró cariograma normal en gemelo $\mathrm{B}$ con restricción del crecimiento (cariotipo 46 xx y complemento cromosómico normal). Entre las semanas 20-25 hay un crecimiento del gemelar A a $816 \mathrm{~g}$ y del gemelar B a $412 \mathrm{~g}$, manteniendo discordancia ponderal de $50 \%$, con doppler normal.

La biometría y el crecimiento ponderal se presentan en Tabla I y el doppler cerebral del gemelar B en semana 26 en Figura 3 . Se suspendió ASA y

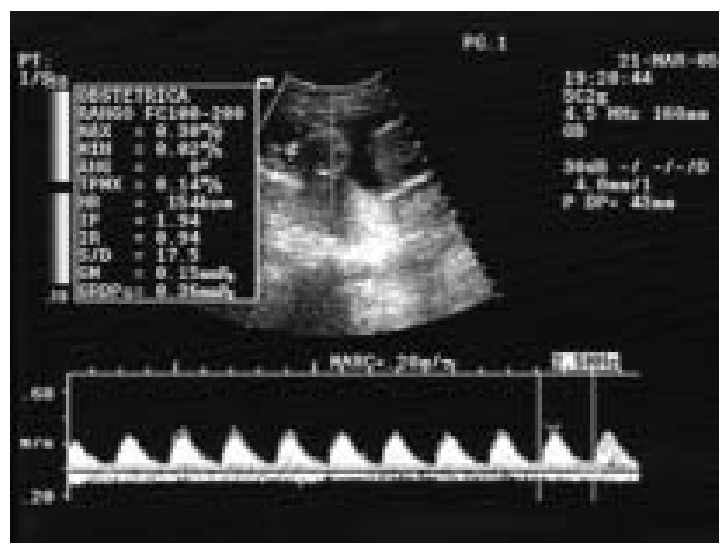

Figura 2. Doppler de arteria umbilical de gemelar B en semana 19 con IP 1,94. 
Tabla I

EVOLUCIÓN DE LA BIOMETRIA DE LOS GEMELOS

\begin{tabular}{|c|c|c|c|c|c|c|c|c|}
\hline \multirow[t]{2}{*}{ Edad gestacional (sem) } & \multicolumn{2}{|c|}{19} & \multicolumn{2}{|c|}{22} & \multicolumn{2}{|c|}{25} & \multicolumn{2}{|c|}{27} \\
\hline & $G A$ & $G B$ & $G A$ & $G B$ & $G A$ & $G B$ & $G A$ & $G B$ \\
\hline Diámetro Biparietal & 46 & 38 & 57 & 48 & 66 & 57 & 69 & 57 \\
\hline Circ. Craneana & 170 & 137 & 212 & 178 & 236 & 209 & 246 & 208 \\
\hline Circ. Abdominal & 151 & 121 & 190 & 141 & 205 & 170 & 218 & 166 \\
\hline Longitud Fémur & 36 & 21 & 40 & 30 & 45 & 36 & 47 & 36 \\
\hline Peso $(g)$ & 358 & 172 & 556 & 275 & 816 & 412 & 880 & 410 \\
\hline Discordancia (\%) & - & 50 & - & 51 & - & 50 & - & 50 \\
\hline
\end{tabular}

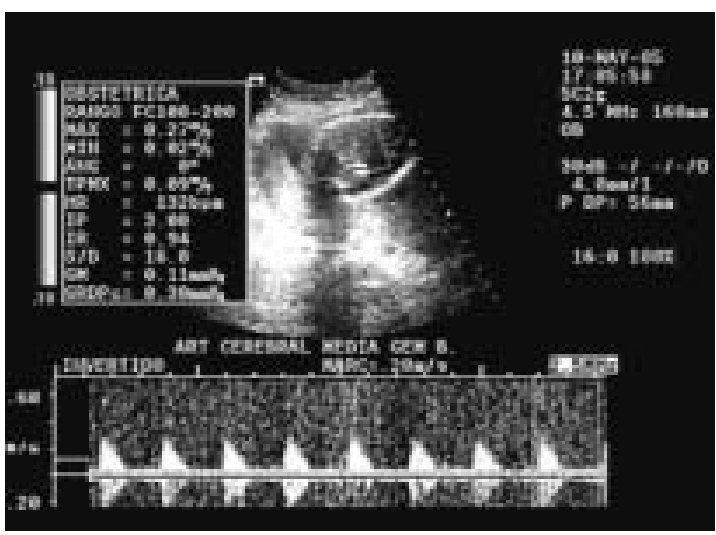

Figura 3. Doppler de arteria cerebral media de gemelar B en semana 26, con IP 3,00.

se indicó corticoides en prevención de distrés respiratorio abriéndose ventana de observación en semana 26-27 en la expectativa de alcanzar peso de sobrevivencia en gemelar B. A las 27 semanas la paciente es hospitalizada por inicio de visión borrosa de ojo derecho, hipertensión arterial (145/ 90 ), hiperreflexia rotuliana y livido reticularis palmar, sin cefalea o edemas; el US confirmó detención del crecimiento de gemelar B (igual peso que semana 25).

Al ingreso destaca trombocitopenia de 60.000/ $\mathrm{mm}^{3}$ y $45.000 / \mathrm{mm}^{3}$ a las 8 horas, albuminuria de 2,1 g/L, LDH $403 \mathrm{U} / \mathrm{L}$, anemia, alteración moderada de pruebas hepáticas, anti-Beta 2 glicoproteína IgG 33,1 e lgM 3,8 U/ml (positivo > 20) (Tabla II). En el período anteparto de 5 días presentó presión arterial normal o alzas moderadas con tres determinaciones de 91-93 de diastólica, hiperreflexia aislada y detectable solo en dos oportunidades en control frecuente, sin edemas.

Se trató con inmunoglobulina $\mathrm{G} 0,4 \mathrm{~g} / \mathrm{kg} / \mathrm{día}$ e.v. por 5 días con recuperación del recuento plaquetario (Figura 4). EI US fetal confirmó restric-
Tabla II

\section{EXÁMENES DE LABORATORIO MATERNO AL INGRESO}

\begin{tabular}{|c|c|c|c|}
\hline Plaquetas & 45.000 & $\mathrm{x} \mathrm{mm}^{3}$ & \\
\hline Albuminuria & 2.102 & $\mathrm{mg} / \mathrm{dl}$ & \\
\hline LDH & 403 & $\mathrm{U} / \mathrm{L}$ & (R: 135-214) \\
\hline Transaminasa GPT & 66 & $\mathrm{U} / \mathrm{L}$ & \\
\hline Transaminasa GOT & 54 & $\mathrm{U} / \mathrm{L}$ & $(\mathrm{R}<31)$ \\
\hline Bilirrubina total & 0,4 & $\mathrm{mg} / \mathrm{dl}$ & \\
\hline Bilirrubina directa & 0,12 & $\mathrm{mg} / \mathrm{dl}$ & \\
\hline Trombina & 30,3 & seg & $(\mathrm{N} \mathrm{23,9)}$ \\
\hline Tiempo de protrombina & 82,1 & seg & (N 27-46) \\
\hline Dímero D & 0,96 & $\mu \mathrm{g} / \mathrm{ml}$ & $(\mathrm{N}<0,5)$ \\
\hline Hematocrito & 31 & $\%$ & \\
\hline Hemoglobina & 9,82 & $\mathrm{mg} / \mathrm{dl}$ & \\
\hline$A A C \lg G$ & 31,1 & $\mathrm{U} / \mathrm{ml}$ & $(+$ Mod 0,1-80) \\
\hline AAC IgM & 6,0 & $\mathrm{U} / \mathrm{ml}$ & $(+$ Mod 20,1-80) \\
\hline $\begin{array}{l}\text { Anti-Beta } 2 \text { glicopro- } \\
\text { teína IgG }\end{array}$ & 33,1 & $\mathrm{U} / \mathrm{ml}$ & \\
\hline $\begin{array}{l}\text { Anti-Beta } 2 \text { glico- } \\
\text { proteína IgM }\end{array}$ & 3,8 & $\mathrm{U} / \mathrm{ml}$ & (Positivo >20) \\
\hline Ácido Úrico & 6,2 & $\mathrm{mg} / \mathrm{dl}$ & \\
\hline Nitrógeno uréico & 15 & $\mathrm{mg} / \mathrm{dl}$ & \\
\hline Anticuerpos Anti ENA & & & Negativos \\
\hline
\end{tabular}

ción severa del gemelar B, con vasodilatación cerebral y se decide interrupción del embarazo.

La cesárea, con precauciones hemostáticas y transfusión plaquetaria intraoperatoria, fue con histerotomía vertical segmento-corporal por presentación gemelar nalgas-tronco, con gemelar B en situación alta y oligoamnios, requiriendo dos maniobras de gran extracción, sin complicaciones. Se obtiene gemelo A femenino, peso $862 \mathrm{~g}$, Apgar 5-8, y gemelar B femenino, peso $408 \mathrm{~g}$, Apgar 88 , ambos $\sin$ hipoxia ni acidosis respiratoria o metabólica. Placenta de $278 \mathrm{~g}$, bicorial-biamniótica fusionada, con cordón de inserción paracentral en la de tamaño mayor y marginal en la de tamaño menor (Figuras 5 y 6). 


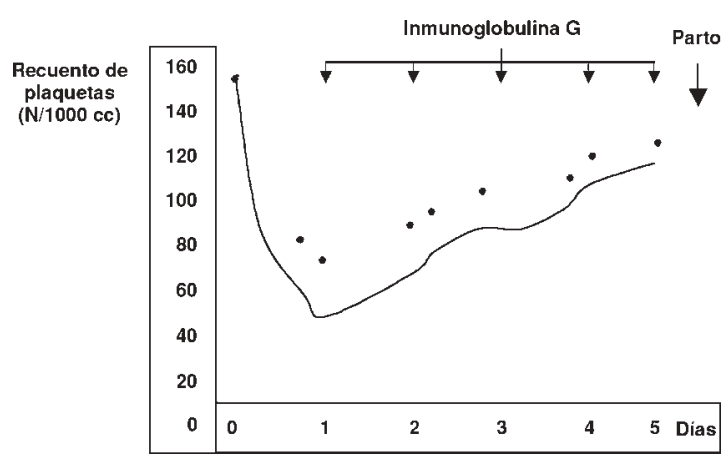

Figura 4. Evolución de la trombocitopenia materna tratada con inmunoglobulina $\mathrm{G}$.

La evolución materna fue de recuperación rápida en sus parámetros clínicos y de laboratorio, con mejoría más lenta de la visión del ojo derecho, que requirió veinte días. La evolución de los gemelos prematuros fue favorable, el A egresó a los 60 días con peso de $2.100 \mathrm{~g}$, y el B egresó a los 100 días con peso de $2.300 \mathrm{~g}$, ambos en muy buenas condiciones.

\section{DISCUSIÓN}

El caso presentado corresponde a un embarazo programado en una paciente con SAF conocido, con antecedente de mortinato, en tratamiento anticoagulante prolongado por cinco años y en condiciones asumidas como óptimas para una nueva gestación.

La HBPM ha demostrado in vitro un efecto favorable sobre el desarrollo del trofoblasto, bloqueando tanto el enlace de AAC con los fosfo-

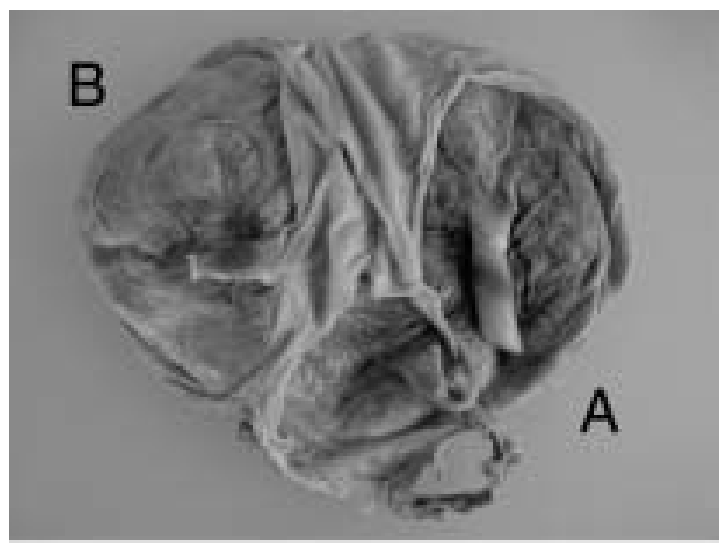

Figura 5. Cara fetal placentas fusionadas y asimétricas. A: gemelar A. B: gemelar B.

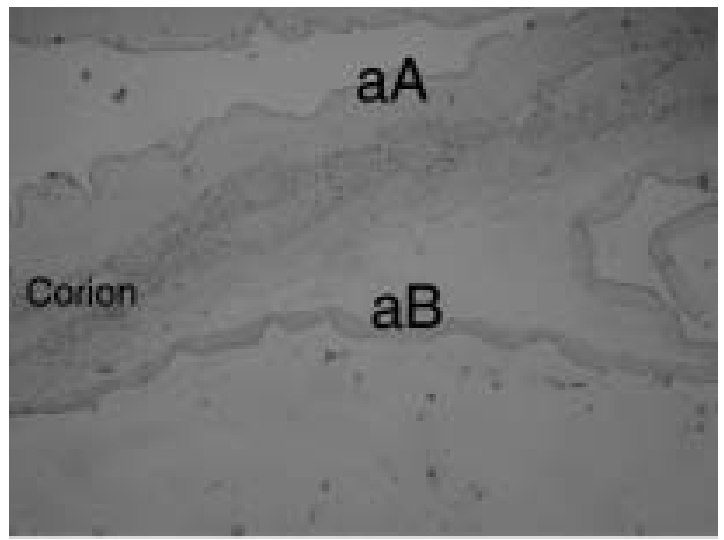

Figura 6. Corte histológico de membranas septales diamnióticas-dicoriónicas fusionadas (H\&E 10X). aA: amnios gemelar A. aB: amnios gemelar B.

lípidos de membrana, como su efecto inhibitorio de invasividad $(13,14)$, y su uso clínico previene la trombosis placentaria $(2,15,16)$. El uso de ASA asociada ha mejorado el pronóstico en pacientes de riesgo (historia de preeclampsia o mortinatos) (15). La HBPM se mantuvo hasta 12 horas antes del parto y ASA fue suspendida en la semana 25 ante la posibilidad de interrupción próxima del embarazo y riesgo aditivo de desprendimiento en gemelar.

La anticoagulación profiláctica fue efectiva con factor Anti $X$ en rango esperado $(0,52$ en semana 12 y 0,43 en semana 28), pero la complicación materna (trombocitopenia) aparece a la misma edad gestacional que la muerte fetal del primer embarazo y se explica porque el SAF, como enfermedad autoinmune tiene evolución cíclica y el tratamiento con HBPM, que evita trombosis, no es curativo ni hace desaparecer los anticuerpos. Clínicamente este tratamiento previene la pérdida reproductiva temprana y mejora la sobrevivencia neonatal $(9,15)$, pero no previene complicaciones maternas más tardías $(10,18,19)$ lo que sugiere que el esquema HBPM-ASA en tercer trimestre y en gemelar aún requiere investigación.

En el caso presentado, a 60 días post-parto y pese al tratamiento anticoagulante prolongado, los AAC e inhibidor lúpico persisten positivos (IgG 56,2 UGPL e IgM 11,1 UMPL, TTPK 108 segundos, protrombina $72 \%$ y plaquetas de 119.000 por $\mathrm{mm}^{3}$ ) demostrando su alta tendencia trombótica.

La complicación en semana 26 sugerente inicialmente de síndrome de HELLP incompleto, sin hipertensión abierta, sin hemólisis ni ictericia, alteración moderada de LDH y enzimas hepáticas, fue mejor interpretada como secundaria a SAF con 
disfunción inmunológica, manifestado a nivel hematológico (trombocitopenia), encefálico (trastorno visual e hiperreflexia aislada), renal (albuminuria e hiperuricemia), piel (livido reticularis palmar) y apoyada en elevación de AAC y presencia de anticoagulante lúpico.

La recuperación plaquetaria con gamaglobulina que bloqueó el sistema retículoendotelial, confirmó su origen inmunológico y permitió la interrupción segura del embarazo. La evidencia sugiere que la trombocitopenia es autoinmune, que se asocia y forma parte del cuadro clínico del SAF permitiendo sospecharlo, pero no incluido en sus criterios diagnósticos (1).

La gemelaridad bicoriónica presentó tres problemas de pronóstico y manejo: 1) restricción temprana del gemelo $B, 2$ ) interpretación del doppler en la exploración hemodinámica y 3 ) decisión del momento de interrupción con prematurez iatrogénica del gemelar $\mathrm{A}$.

1) La restricción generó desde la semana 19 discordancia de peso del $50 \%$, variable independiente de riesgo, de mal pronóstico perinatal (20) y detención definitiva en la semana 25 con peso fetal de $400 \mathrm{~g}$, de viabilidad muy improbable, lo que se asocia más frecuentemente a malformación o genopatía, hipótesis descartadas por US seriado y estudio cromosómico en gemelar B. El SAF produce restricción por vasculopatía vellositaria o trombosis placentaria (9), pero la anticoagulación prolongada (preconcepcional y antenatal) y el doppler normal de arterias uterinas hacían improbable esta explicación para la restricción selectiva. Excluidas otras causales como monocorionicidad, hipertensión, infección, ambiental o drogas, anomalía mülleriana, su origen se orientó a un factor primario, fetal o placentario, que afectó solo al gemelo B y cuya naturaleza se definiría en el nacimiento. Las placentas fueron dicoriónicas fusionadas, con la B más pequeña e isquémica, cordón marginal y vasos umbilicales cercanos al borde placentario. La fusión detiene la expansión de la más pequeña determinando menor área de intercambio y menor peso de nacimiento (21), pero la evolución de la restricción sugiere que esta explicación es insuficiente. La fusión en dicigotos no influye en el peso, pero un monocigoto dicoriónico con placenta fusionada, pesa en promedio $300 \mathrm{~g}$ menos que uno con placentas separadas y el $60 \%$ tienen inserción funicular excéntrica (21). Una anastomosis intraplacentaria, rarísima en dicoriónicos (22), fue descartada con inyección láctea funicular postparto. Dado que ambos gemelos son femeninos con igual grupo sanguíneo (II-
$A, R h+)$, es posible que sean monocigotos, en cuyo caso un mecanismo embriológico postcigótico temprano, de distribución desigual de blastómeras desde el cigoto original, con menor alocación de células en el embrión B (23) explicaría su menor tamaño, la discordancia precoz en gemelos genéticamente iguales y el efecto de la fusión placentaria. Este mecanismo en monocigotos, confirmado en un subgrupo de enfermedades genéticas ligadas al cromosoma $X(24,25,26,27)$ se ha postulado para explicar el gemelar evanescente, el papiráceo, el acardio y la discordancia por malformaciones o por crecimiento. El estudio de cigocidad es importante en la etiopatogenia y el pronóstico (28). El caso presentado sería una restricción primaria de origen embriológico del gemelo $\mathrm{B}$, con menor celularidad y constitucionalmente pequeño, combinada con una restricción hipóxica secundaria después de la semana 20 , originada en la insuficiencia de una placenta fusionada, más pequeña y menor área de intercambio. La angiogénesis placentaria es modulada por el flujo uterino, la presión parcial de oxígeno en el espacio intervelloso y factores moleculares que permiten adaptar el desarrollo de vasos vellosos a un régimen de baja resistencia para abastecer las demandas del crecimiento (29). En la adaptación circulatoria hasta la semana 25, puede especularse un rol protector de la placenta del gemelo A sobre su cogemelo, a través de sus propios factores angiogénicos.

2) El estudio hemodinámico con doppler presentó flujo de arterias uterinas materna normal, y en el gemelo $B$ flujo en arteria umbilical de inicio tardío e intermitente en semana 19 y luego normal estable hasta el parto, vasodilatación cerebral en semana 28 y ductus venoso normal, hallazgos diferentes a lo esperado en restricción severa que debieron ser explicados adecuadamente para un manejo apropiado.

Doppler uterino: la resistencia uterina y su valor predictivo en gemelos son más bajos que en únicos por influencia de la mayor área de invasión trofoblástica biplacentaria $(30,31)$. En el caso, el efecto sobre la resistencia uterina de la invasión focalmente defectuosa de la placenta B, fue atenuado por la implantación adecuada de la placenta A y su posición, más baja y cercana a las arterias uterinas, que favoreció su representación en el índice. Pese al doppler normal puede haber complicaciones posteriores, porque su incidencia es significativamente mayor que en únicos y la interpretación descrita no resta valor al método ya que es el de mayor sensibilidad en la evaluación de la circulación uteroplacentaria. 
Doppler umbilical del gemelo B: su aparición tardía (semana 19) y normalidad posterior significa angiogénesis vellosa adecuada para sostener el crecimiento de un feto primariamente más pequeño con menor demanda, es propia de los pequeños constitucionales $(32,33,34)$. Posteriormente, en semana 26-27, etapa de remodelación fisiológica en la angiogénesis $(35,36,37)$ con elongación de capilares para abastecer mayor demanda, el feto detiene su crecimiento, influyendo la menor área de intercambio de la placenta B y la vasculopatía evidenciada por menor desarrollo velloso y capilar (Figuras 7 y 8 ). Esto indica el inicio de hipoxia placentaria (38), sobrepuesta a la restricción primaria siendo transitoria la normalidad observada del doppler umbilical. La progresión esperada a flujo ausente o reverso es un signo de hipoxia más grave y tardía que se asocia con mortalidad perinatal; en cambio en el caso presentado, asociada al manejo (interrupción), se correlacionó con nacimiento sin asfixia y sobrevivencia del gemelar B. Una clasificación clínica de gravedad de restricción bajo percentil 10, según frecuencia cardiaca fetal (FCF) e índice de pulsatibilidad umbilical (IP), demostró que casos menos graves con FCF e IP normales, independiente de su peso, nacen sin hipoxia ni acidosis, a diferencia de similares con alteración de ambos índices (39), lo cuál es concordante con lo observado.

Vasodilatación en arteria cerebral media del gemelo B: signo precoz de adaptación en la secuencia hemodinámica de la restricción $(40,41)$ cuya reversión puede continuarse con muerte fetal y se utiliza para decidir oportunamente la interrupción. En gemelar B, su hallazgo en semana 28 indicó redistribución circulatoria local reciente e indemnidad del mecanismo autorregulatorio cerebral, para mantener perfusión, ante imposibilidad de mayor oxigenación desde el espacio intervelloso. La vasodilatación cerebral antenatal en seguimiento de largo plazo de neonatos con restricción se asocia a indemnidad neurológica $(42,43,44)$ y además en el caso no hubo asfixia periparto ni lesiones centrales (hemorragia, cavitarias o ecodensidades) con buena respuesta al manejo nutricional, sosteniendo temporalmente un pronóstico prudente del gemelar B. El ductus venoso normal significa que no hubo disfunción miocárdica, y es importante porque el monitoreo solamente del lado arterial del feto en riesgo vital es insuficiente para detectar un mayor deterioro $(45,46)$.

3) Interrupción: lo óptimo en restricción del crecimiento fetal antes de la semana 32 , debe ba-

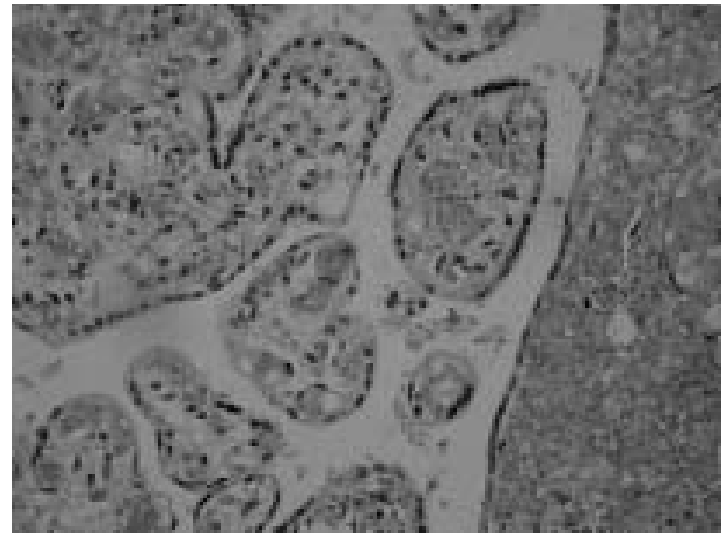

Figura 7. Microscopía de placenta de gemelar A. Se observan vellosidades congestivas y aumentadas de tamaño (H\&E $40 \mathrm{X}$ ).

lancear los riesgos de la prematurez, contra exposición fetal prolongada a hipoxemia. Están en estudio los métodos de evaluación con doppler arterial y venoso, perfil biofísico y FCF (estudio TRUFFLE), cuyas modificaciones relacionadas al pronóstico determinarán la mejor oportunidad del parto. La indicación de interrupción en semana 28 por causa materna, con adaptación circulatoria inicial en el gemelo B, incluyó el concepto de menor tasa de complicaciones por cada semana ganada antes de interrumpir $(47,48)$, protegiendo además el pronóstico del gemelo sano. La sobrevida actual de prematuros con 28 semanas es alta, pero su asociación a un peso de $408 \mathrm{~g}$ es una situación infrecuente e inédita en nuestro medio. El resultado, sugerente de que la madurez es más impor-

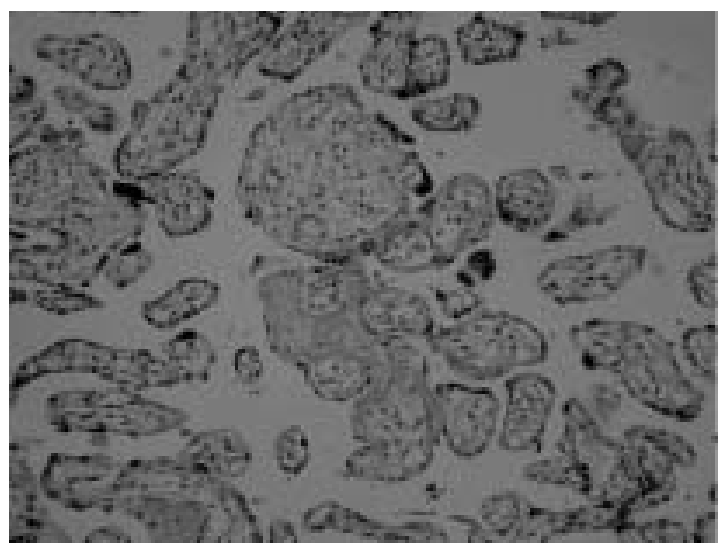

Figura 8. Microscopía de placenta de gemelar B. Se observan vellosidades pequeñas con escasa fibrosis estromal y fibrina (H\&E 40X). 
tante en la sobrevivencia que el peso, tiene consecuencias médicas y éticas. De las médicas, la más importante es el manejo nutricional intensivo prolongado, porque expone al neonato a complicaciones propias y a la respuesta inflamatoria, posibilitando secuelas de mediano y largo plazo. Las consecuencias éticas son que el tratamiento intensivo de un prematuro de bajo peso extremo, en el contexto descrito, es una terapia proporcionada al problema y su indicación es preferencial y no opcional, y que el concepto empírico de "límite de viabilidad" determinado solo por el peso es limitado y su aplicación sin considerar otros factores tiene el sesgo de no tratar casos viables.

\section{CONCLUSIONES}

1. En un caso complejo por asociación de SAF complicado y gemelar con restricción grave, se logró sobrevivencia perinatal de ambos gemelos.

2. El resultado se relaciona con los mecanismos adaptativos fetales y neonatales de enfermedad y con el manejo de las complicaciones maternofetales.

3. La interrupción del embarazo previno la muerte de un gemelo de muy bajo peso y generó prematurez iatrogénica en un gemelo sano.

4. La interpretación definitiva de la restricción grave y su hemodinamia en un gemelo requiere identificar su cigocidad.

5. El manejo de casos complejos debe efectuarse en unidades especializadas.

6. La cronología de la gemelaridad es una variable importante del estudio de gemelos.

\section{BIBLIOGRAFÍA}

1. Galli M, Barbui T. Antiphospholipid syndrome: Definition and treatment. Semin Tromb Hemost 2003;29(2):195-203.

2. Carp HJ. Antiphospholipid síndrome in pregnancy. Curr Opin Obstet Gynecol 2004;16(2):129-35.

3. Asherson RA. The catastrophic antiphospholipid (Asherson`s) syndrome in 2004 - a review. Autoimmun Rev 2005;4(1):48-54 .

4. Le Thi Thuong D, Tieulie N, Costedoat N, Andreu MR, Wechsler B, Vauthier-Brouzes D, et al. The HELLP syndrome in the antiphospholipid syndrome: retrospective study of 16 cases in 15 women. Ann Rheum Dis 2005;64(2):273-8.

5. Gibson GE, Su WP, Pittelkow MR. Antiphospholipid syndrome and the skin. J Am Acad Dermatol 1997;36:970-82.

6. Cruz D. Renal manifestations of the antiphospholipid syndrome. Lupus 2005; 14(1):45-8.
7. Bas de Laat H, Derksen R, G. De Groot P. Beta2Glycoprotein I, the playmaker of the antiphospholipid syndrome. Clin Immunol 2004;112:161-8.

8. Mackworth-Young. Antiphosphlipid síndrome: multiple mechanisms. Clin Exp Inmunol 2004;136:393401.

9. Sebire N J, Backos M, El Gaddal S, Goldin RD, Regan L. Placental pathology, antiphospholipid antibodies and pregnancy outcome in recurrent miscarriage patients. Obstet Gynecol 2003;101:258-63.

10. Branch DW, Scott JR, Kochenour NK, Hershgold E. Obstetric complications associated with the lupus anticoagulant. N England J Med 1986;313(21),13226.

11. Tartakovsky B, Bermas BL, Sthoeger Z, Shearer GM, Mozes E. Defective maternal-fetal interaction in a murine autoimmune model. Hum Reprod 1996;11: 2408-11.

12. Sthoeger Z, Mozes E, Tartakovsky B. Anti-cardiolipin antibodies induce pregnancy failure by impairing embryonic implantation. Proc Natl Acad Sci USA 1993;90(14):6464-7.

13. Quenby S, Mountfield, Cartwright J, Whitley G, Vince G. Effects of low-molecular weight and unfractionated heparin on trophoblast function. Obstet Gynecol 2004;104:3564-1.

14. Di Simone N, Caliandro D, Castellani R, Ferrazzani S, De Carolis S, Mancuso S, Caruso A. Low molecular weight heparine restore in-vitro trophoblast invasiveness and differentiation in presences of inmunoglobulin $\mathrm{G}$ fractions obtained from patients with antiphospholipid syndrome. Hum Reprod 1999; 14:489-95.

15. Rai R, Cohen H, Dave M, Regan L. Randomized controlled trial of aspirin and aspirin plus heparin in pregnant women with recurrent miscarriage associated with phospholipid antibodies (or antiphospholipid antibodies) BMJ 1997;314:253-7.

16. Lassere M, Empson M. Treatment of antiphospholipid syndrome in pregnancy - a systematic review of randomized therapeutic trials. Tromb Res 2004;114: 419-26.

17. Cervera R, Balasch J. The management of pregnant patients with antiphospholipid syndrome. Lupus 2004;13(9):683-7.

18. Carbillon L, Sauvet ML, Fain O, Aurousseau. Letter to Editor. Treated pregnant patients with antiphospholipid syndrome are particularly susceptible to preeclampsia and fetal growth retardation. J Reprod Immunol 2005;65:89-90.

19. Bakos M, Rai R, Baxter N, Chilcott IT, Cohen H, Regan L. Pregnancy complications in women with recurrent miscarriage associated with antiphospholipid antibodies treated with low dose heparin and aspirin . Br J Obstet Gynaecol 1999;106:102-7.

20. Vergani P, Locatelli M, Scian A, Pozzi E, Pezullo J, Ghidini A. Preterm twins: what threshold of birth weight discordance heralds major adverse neonatal outcome? Am J Obstet Gynecol 2004;191:1441-5. 
21. Loos RJF, Derom C, Derom R, Vlietinck R. Birthweight in liveborn twins: the influence of the umbilical cord insertion and fusion of placentas. $\mathrm{Br} \mathrm{J}$ Obstet Gynaecol 2001;108:943-8.

22. Lage JM, Vanmarter LJ, Mikhail E. Vascular anastomoses in fused, dichorionic twin placentas resulting in twin transfusion syndrome. Placenta 1989;10:559.

23. Machin G, Bamforth F, Innes Micheil, McNichol K. Some perinatal characteristics of monozygotic twins who are dichorionic. Am J Med Genet 1995;55:71-6.

24. Puck JM. The timing of twinning: More insight from X inactivation. Am J Hum Genet 1998;63:327-8.

25. Plenge RM, Stevenson RA, Lubs HA, Schwartz CE, Willard HF. Skewed X-chromosome inactivation is a common feature of $\mathrm{X}$-linked mental retardation disorders. Am J Hum Genet 2002;71:168-73.

26. Monteiro J, Derom C, Vlietinck R, Kohn N, Lesser $\mathrm{M}$, Gregersen PK. Commitment to inactivation precedes the twining event in monochorionic MZ twins. Am J Hum Genet 1998;63:339-46.

27. Grennert L, Persson PH, Genner G, Gullberg B. Zygosity and intrauterine growth of twins. Obstet Gynecol1980;55(6):684-7.

28. Loos R, Derom C, Vlietink R, Derom R. The East Flanders Prospective Twin Survey (Belgium): a population-based register. Twin Res 1998;1:16775.

29. Regnault TRH, de Vrijer B, Galan HL, Davidsen ML, Trembler KA, Battaglia FC, et al. The relationship between transplacental $\mathrm{O} 2$ diffusion and placental expression of PIGF, VEGF and their receptors in a placental insufficiency model of fetal growth restriction. J Physiol 2003;550(2):641-56.

30. Yu CKH, Papageorghiou AT, Boli A, Cacho AM, Nikolaides $\mathrm{KH}$. Screening for pre-eclampsia and fetal growth restriction in twin pregnancies at 23 weeks of gestation by transvaginal uterine artery Doppler. Ultrasound Obstet Gynecol 2002;20(6): 535-40.

31. Geipel A, Berg C, Germer U, Katalinic A, Krapp M, Smrcek J, Gembruch U. Doppler assessment of the uterine circulation in the second trimester in twin pregnancies: prediction of pre-eclampsia, fetal growth restriction and birth weight discordance. Ultrasound Obstet Gynecol 2002;20(6):541-5.

32. McCowan LME, Harding JE, Robert AB, Barker SE, Ford C, Stewart AW. A pilot randomized controlled trial of two regimens of fetal surveillance for smallfor-gestational age fetuses with normal results of umbilical artery Doppler velocimetry. Am J Obstet Gynecol 2000;182:81-6.

33. Dase J, Mclntire D, Lucas M, Leveno K. Effects of symmetric and asymmetric fetal growth on pregnancy outcomes. Obstet Gynecol 2000;96:321-7.

34. Severy FM, Bocchi C, Visentin A, Falco P, Cobellis $\mathrm{L}$, Florio $\mathrm{P}$, et al. Uterine and fetal cerebral Doppler predict the outcome of third-trimester small-for- gestational age fetuses with normal umbilical artery Doppler. Ultrasound Obstet Gynecol 2002;19:225-8.

35. Kaufmann P, Mayhew TM, Charnock-Jones DS. Aspects of human fetoplacental vasculogenesis and angiogenesis. II. Changes during normal pregnancy. Placenta 2004;25:114-26.

36. Mayhew TM, Charnock-Jones DS, Kaufmann P. Aspects of human fetoplacental vasculogenesis and angiogenesis. III. Changes in complicated pregnancies. Placenta 2004;9(25):127-39.

37. Macara L, Kingdom JCP, Kaufmann P, Konen G, Hair J, More I, et al. Structural analysis of placental terminal villi from growth-restricted pregnancies with abnormal umbilical artery doppler waveforms. Placenta 1996;17:37-48.

38. Kingdom JCP, Kaufmann P. Oxigen and placental villous development: origins of fetal hypoxia. Placenta $1997 ; 18: 613-21$.

39. Pardi G, Marconi AM, Cetin N. Placental- Fetal Interrelationship in IUGR fetuses-a review. Placenta 2002; 23 Suppl A:S136-41.

40. Bashat A, Genbruch U, Harman C. The sequence of changes in Doppler and biophysical parameters as severe fetal growth restriction worsens. Ultrasound Obstet Gynecol 2001;18:571-7.

41. Johnson P, Stojilkovic T, Sarkar P. Middle cerebral artery Doppler in severe intrauterine growth restriction. Ultrasound Obstet Gynecol 2001;17(5):416-20.

42. Scherjon SA, Oosting $H$, Smolders-DeHaas $H$, Zondervan HA, Kok JH. Neurodevelopment outcome at three years of age after fetal "brain-sparing". Early Hum Dev 1998;52(1):67-79.

43. Scherjon SA, Smolders-DeHaas H, Kok JH, Zondervan HA. The "brain-sparing" effects: antenatal cerebral Doppler findings in relation to neurologic outcome in very preterm infants. Am $\mathrm{J}$ Obstet Gynecol 1993;169(1):169-75.

44. Chan FY, Pun TC, Lam YH. Fetal cerebral Doppler studies as a predictor of perinatal outcome and subsequent neurologic handicap. Obstet Gynecol 1996;87:981-8.

45. Caruso A, De Carolis S, Ferrazzani S, Valesini G, Caforio L, Mancuso S. Pregnancy outcome in relation to uterine artery flow velocity waveforms and clinical characteristics in women with antiphospholipid syndrome. Obstet Gynecol 1993;82:970-7.

46. Kiserud T, Acharya G. The Fetal Circulation. Prenat Diagn 2004;24:1049-59.

47. Bellotti M, Pennati G, De Gasperi C, Frederick C, Bataglia, Ferrazi E. Role of ductus venosus in distribution of umbilical blood flow in human fetuses during second half of pregnancy. Am J Physiol Heart Circ Physiol 2000;279(3):H1256-63.

48. Bilardo $\mathrm{CM}$, Wolf $\mathrm{H}$, Stigter $\mathrm{RH}$, Ville $\mathrm{Y}$, Baez $\mathrm{E}$, Visser GHA, et al. Relation between monitoring parameters and perinatal outcome in severe, early intrauterine growth restriction. Ultrasound Obstet Gynecol 2004;23:119-25. 